\title{
IMPACT DU BRAND CONTENT SUR LES MEDIAS SOCIAUX
}

\author{
EL MOKHTARI Soufiane $^{\mathbf{1}}$, OUIDDAD Smail ${ }^{2}$, and JEDDIOUI Aboubakr ${ }^{3}$ \\ ${ }^{1}$ University HASSAN First, ENCG, SETTAT, MAROC \\ ${ }^{2}$ University HASSAN First, ENCG, SETTAT, MAROC \\ ${ }^{3}$ Institut Supérieur De Commerce et d'Administration Des Entreprises, MAROC
}

\begin{abstract}
Résumé : Cet article, qui s'inscrit dans le cadre du marketing des médias sociaux, s'intéresser aux caractéristiques du Brand Content qui ont été explorées dans la littérature en tant que facteurs potentiels ayant un impact sur l'engagement des consommateurs, nous allons présenter les objectifs, la méthodologie et l'analyse bibliométrique de la revue de littérature systématique sur la base de 44 articles en vue de mesurer des variables explicatives impactant l'engagement des consommateurs sur les médias sociaux.
\end{abstract}

Mots-clés : Marketing des médias sociaux - Brand Content - Engagement des consommateurs.

\begin{abstract}
This article, which belongs to the marketing framework of social media, focuses on the characteristics of branded content that were explored in literature as potential factors impacting consumer engagement. We will present a systematic literature review basing on 44 articles so as to measure the explainable differences impacting consumers 'engagement on social
\end{abstract}

Keywords: Social media Marketing-Brand content-consumer engagement.

\section{INTRODUCTION}

L'avènement des plateformes des médias sociaux durant les 20 dernières années et l'adoption en croissance continue de ces plateformes par les consommateurs ont précipité un changement de paradigme et ont impacté significativement la manière dont ces consommateurs communiquent et interagissent entre eux et avec les marques. La nature interactive des médias sociaux a donné plus de pouvoir aux consommateurs en les transformant de récepteurs passifs aux messages publicitaires à des participants actifs pouvant exprimer leur satisfaction ou mécontentement face à un produit, soutenir ou dénigrer une marque, partager ou critiquer une publicité.

Devenant conscientes de l'enjeu et du potentiel des médias sociaux pour toucher une plus grande audience à un coût plus faible tout en engageant les consommateurs dans une communication bidirectionnelle, les marques n'avaient pas le choix que d'adopter des stratégies Marketing spécifiques aux médias sociaux. On assiste dès lors à l'apparition d'une nouvelle discipline (SMM : Social Media Marketing) dont le fer de lance sera le Brand Content. La marque prend ainsi une nouvelle posture d'éditrice de contenu proposant des tutoriels, des recettes, des web séries en plusieurs épisodes, des astuces et des conseils en espérant attirer l'attention et faire interagir un 
consommateur de plus en plus sélectif face au grand volume de messages publicitaires qui l'entourent.

En effet, la recherche académique appelle de plus en plus à des etudes approfondies afin de déterminer pourquoi un Brand Content donné serait engageant sur les médias sociaux et pourquoi un autre ne le serait pas (Kumar, 2015 ; Marketing Science Institute, 2018). Ainsi, notre article de recherche s'intéresse spécifiquement à ce champ de recherche, nous ambitionnons déjà d'identifier les articles académiques ayant essayé d'apporter une réponse à cette question afin de mieux cerner l'état des connaissances. Dans le présent article, nous nous intéressons à explorer les variables qui ont été étudiées par les chercheurs comme variables explicatives, dépendantes de l'engagement sur les médias sociaux. Ainsi, nous nous sommes fixés comme objectifs de répondre à la question suivante : Quelles sont les variables explicatives du brand content qui impactent sur les médias sociaux.

Pour répondre à cette question nous allons mener une revue de littérature systématique sur la base de 44 articles de recherché indexé dans google scholar.

\section{RESULTAT DE LA REVUE DE LITTERATURE}

Sur les 44 articles de notre revue de littérature, 11 variables ont été étudiées uniquement comme des variables explicatives.

Tableau 1. Les variables explicatives étudiées par les articles de notre revue

\begin{tabular}{|l|c|}
\hline Variable explicative & $\begin{array}{c}\text { Nombre } \\
\text { d'articles }\end{array}$ \\
\hline Informativité (Informativeness) & 19 \\
\hline Émotivité (Emotionality) & 19 \\
\hline Catégorie de contenu (Content category) & 13 \\
\hline Interactivité (Interactivity) & 12 \\
\hline Contenu rémunératif et promotionnel (Remuneration \& Promotions) & 12 \\
\hline Vivacité (Vividness) & 11 \\
\hline Divertissement (Entertainment) & 8 \\
\hline Degré de présence de la marque (Brand Presence) & 7 \\
\hline Cause sociale (Social responsibility content) & 4 \\
\hline Distinctivité et nouveauté du contenu (Distinctiveness and Novelty) & 2 \\
\hline Valence des commentaires (Comments Valence) & 2 \\
\hline
\end{tabular}

\section{A. Informativité du contenu}

Selon De Vries et al. (2012), le contenu informatif contient des informations sur des produits, des marques, des entreprises ou des activités marketing spécifiques. Le contenu informatif des publications informe les utilisateurs des alternatives de produits, leur permettant ainsi de faire de meilleurs choix (De Vries et al., 2012). Si la publication d'une marque contient des informations telles que les dates de lancement des nouvelles versions et la description des produits, les utilisateurs seront motivés pour interagir et pour 
consommer (De Vries et al., 2012). Si les utilisateurs sont motivés, ils seront susceptibles de réagir aux publications (Cvijikj et Michahelles, 2011).

\section{B. Emotivité du contenu}

Swani et al. (2013) ont défini le contenu émotionnel d'une marque comme un contenu qui tente de susciter des émotions négatives ou positives chez le public avec des messages contenant des thèmes tels que la peur, l'humour, l'amour, la sensualité, l'aventure, la culpabilité et d'autres indices émotionnels. Akpinar et Berger (2017) ont décrit le contenu émotionnel d'une marque comme un appel affectif conçu pour faire apparaitre les émotions du destinataire en utilisant des stratégies telles que le drame, l'humeur, la musique ou d'autres émotions. Tafesse et Wien (2018) ont défini le contenu émotionnel de marque comme un contenu évoquant les émotions des consommateurs. Ce contenu utilise généralement un langage chargé d'émotions, des histoires inspirantes, de l'humour et des blagues pour susciter des réactions affectives telles que l'amusement, l'excitation, l'émerveillement, etc.

Les thèmes communs de ce type de contenu représentent des publications émotionnellement expressives, des récits émotionnels, des blagues et des anecdotes. Tellis et al. (2018) ont fondé leur définition du contenu émotionnel d'une marque sur une revue approfondie de la littérature publicitaire et ont expliqué que, dans la littérature sur la publicité et sur le contenu d'une marque, les travaux ont évalué l'effet du contenu émotionnel par rapport au contenu informatif, l'émotivité générale du contenu (élevé ou faible), le rôle des émotions discrètes spécifiques et les dimensions pour décrire les émotions.

\section{Catégorie de contenu}

Plusieurs chercheurs se sont intéressés à catégoriser les contenus de marques sur les réseaux sociaux en analysant les pratiques de marques. Cette catégorisation est généralement basée sur le sujet du contenu (Topic). Certains chercheurs se sont intéressés à déduire ces catégories après une analyse des contenus produits par une ou plusieurs marques sur les réseaux sociaux puis ils ont essayé de les classifier en catégories selon le sujet (Cvijikj et al., 2011, Schultz 2016). Schultz (2016) a constaté que les publications Brand Content de jeux concours avaient un impact négatif sur l'engagement des consommateurs sur Facebook. Cette constatation était surprenante pour l'auteur car les publications de jeux concours sur les sites de réseaux sociaux invitent généralement les utilisateurs à aimer, commenter et/ou partager certaines informations.

\section{Intéractivite du contenu}

L'interactivité peut être définie comme le degré auquel deux ou plusieurs interlocuteurs influent l'un sur l'autre sur un support de communication et par des messages, et le degré auquel les actions de ces interlocuteurs sont synchronisées (Liu et Shrum, 2002). Par exemple, les utilisateurs donnent leur avis sur les produits ou répondent aux informations concernant des produits fournis par des vendeurs en ligne. L'interactivité est largement considérée comme un facteur essentiel dans la détermination d'une variété de résultats affectifs et comportementaux tels que la satisfaction, l'attitude, la prise de décision et l'implication (Coyle et Thorson, 2001 ; Fortin et Dholakia, 2005 ; Stewart et Pavlou, 2002).

Un degré élevé d'interactivité peut conduire à des résultats de communication intéressants (Macias, 2003 ; Rafaeli et Ariel, 2007 ; Sicilia et al., 2005). En outre, il a été démontré que les perceptions des utilisateurs concernant l'interactivité étaient positivement liées à leurs attitudes à l'égard de certains sites Web et produits (Sundar et Kim, 2005). Au niveau du contenu, il a été indiqué que l'interactivité varie d'un contenu à l'autre (Ko et al., 2005). 


\section{E. Contenu promotionnel}

Swani et al. (2013) ont défini le Brand Content promotionnel comme des appels directs à des achats faisant référence à des appels explicites encourageant les acheteurs potentiels à effectuer un achat immédiat. Selon ces auteurs, ces appels à l'action pourraient être des recommandations pour effectuer un achat (acheter maintenant, les offres et les promotions se terminent aujourd'hui).

Dans le but d'analyser les facteurs d'influence en termes de caractéristiques du contenu communiqué par les marques par rapport au niveau d'engagement des consommateurs en ligne mesuré par le nombre de mentions 'j'aime', de commentaires et de partages sur Facebook, Cvijikj et Michahelles (2013) ont constaté que le contenu rémunérateur s'écartait du comportement attendu. Bien que l'effet positif n'ait été constaté que sur le ratio des commentaires, aucun effet n'a été constaté sur le ratio des partages.

\section{F. Vivacité du contenu}

Selon Tafesse (2015), la vivacité du contenu est un concept qui définit la richesse représentationnelle d'un environnement médiatisé (Coyle et Thorson, 2001 ; Kaplan et Haenlein, 2010). Les caractéristiques des contenus de marque, y compris la vivacité, ont jusqu'à présent été étudiées avec des résultats mitigés, en raison peut-être de différences dans les méthodes de mesure. Par exemple, certaines études rapportent un effet positif de la vivacité du contenu sur la réaction du public (par exemple, Cvijikj et Michahelles, 2013 ; Sabate et al., 2014), tandis que d'autres font état d'un effet négatif (par exemple, De Vries et al., 2012).

De Vries et al. (2012) ont montré que les publications en ligne très vives recevaient beaucoup plus de mentions 'j'aime' que les publications manquant de vivacité. Cvijikj et Michahelles (2013) ont constaté qu'un Brand Content très vif avait un impact positif sur l'engagement, et plus particulièrement sur les mentions 'j'aime' et les partages par les consommateurs.

\section{G. Degré du divertissement du contenu}

Luarn et al. (2015) ont défini le Brand Content divertissant comme un type de message qui ne fait pas référence à la marque ou à un produit particulier. Les messages de divertissement sont plutôt des vidéos humoristiques, des anecdotes, des slogans ou des jeux de mots (Cvijikj et Michahelles, 2013). Ils offrent aux consommateurs une occasion de se distraire et de se divertir, ainsi que du plaisir esthétique et une libération émotionnelle (Bronstein, 2013 ; Haghirian et al., 2005). Selon ces auteurs, des études antérieures ont montré que le divertissement est le facteur le plus déterminant dans le comportement des utilisateurs sur les réseaux sociaux (Lin et Lu, 2011 ; Sledgianowski et Kulviwat, 2009). Menon et al. (2019) ont décrit le Brand Content divertissant comme des publications qui suscitent l'intérêt des utilisateurs, telles que des images, des photos et des vidéos intéressantes, des anecdotes ou du contenu visant à renseigner les utilisateurs sur des sujets de manière divertissante. Cependant, si le Brand Content divertissant procure du plaisir, il n'est pas nécessaire qu'il soit associé au produit ou à la marque.

\section{H. Degré de presence de la marque}

Alboqami et al. (2015) ont utilisé le terme 'centralité de la marque' pour désigner le degré de présence de la marque dans son contenu publié sur Twitter et indiquer si la marque est au centre du contenu ou non. Selon ces auteurs, il est difficile d'introduire plus d'un sujet dans un même tweet en raison de la limite de 
140 caractères. Par conséquent, la centralité de la marque peut changer en fonction du contenu. Les résultats de l'étude ont révélé un impact significatif de la centralité de la marque sur le comportement de partage.

Selon Akpinar et Berger (2017), avec le marketing des médias sociaux, l'attention s'est déplacée vers les partages. En outre, des recherches ont suggéré que la présence de la marque pourrait réduire les partages (Stephen, Sciandra et Inman 2015 ; Teixeira 2012). Les annonceurs peuvent donc éviter de faire de la marque une partie intégrante de la publicité car le Brand Content peut ressembler davantage à de la publicité sur les réseaux sociaux, ce qui pourrait réduire le nombre de partages (Stephen, Sciandra et Inman 2015).

\section{Cause sociale}

Khan et al. (2016) ont utilisé l'expression 'communication de la responsabilité sociétale des entreprises' et l'ont définie sur la base de la définition précédente de Morsing comme suit : c'est une communication conçue et distribuée par l'entreprise elle-même concernant ses efforts en matière de responsabilité sociétale (RSE). Selon ces auteurs, les entreprises communiquent des messages liés à la RSE pour améliorer l'indice de performance et développer une image responsable (Nielsen et Thomsen, 2012)

\section{J. Distinctivité et nouveauté du contenu}

Dans leur étude visant à évaluer les caractéristiques du Brand Content qui conduisent à la visualisation virale en ligne de la publicité diffusée à la télé, Southgate et al. (2010) ont analysé plus de 100 vidéos postées par des marques au Royaume-Uni et aux États-Unis sur Youtube entre 2007 et 2009 et s'attendaient à ce que seul un nombre limité de vidéos publicitaires télévisées soit suffisamment intéressant pour que les consommateurs puissent les rechercher et les visionner en ligne. Selon ces auteurs, dans cet environnement de visionnage libre, les publicités doivent se démarquer et la nouveauté du contenu joue un rôle important dans l'engagement des consommateurs envers ce contenu en ligne

\section{K. Valence des commentaires}

Plusieurs travaux de recherche ont montré que les discussions en ligne des consommateurs sur des expériences positives de produits ou de marques peuvent générer de l'empathie et des sentiments positifs chez les lecteurs (par exemple, Bickart et Schindler 2001). Cet échange d'informations et d'expériences entre consommateurs a un effet positif sur la perception de la valeur d'un produit, la probabilité de recommander le produit (Gruen, Osmonbekov et Czaplewski 2006) et ainsi sur les ventes (par exemple, Chevalier et Mayzlin 2006 ; Chintagunta, Gopinath et Venkataraman 2010). Sur la base de cette littérature, De Vries et al. (2012) ont étudié la valence des commentaires en tant que caractéristique du Brand Content pouvant avoir un impact sur l'engagement des consommateurs sur les médias sociaux.

\section{CONCLUSION}

Le présent article nous a permis de définir les caractéristiques du Brand Content qui ont fait référence à tous les facteurs d'exécution ainsi que l'ensemble des stratégies du message qui sont utilisés par les marketers pour délivrer leurs contenus aux audiences ciblées. Ces caractéristiques concernent la conception du contenu, l'exécution opérationnelle du message, et incluent la notion de conception des communications de manière à augmenter les chances qu'elles puissent produire les effets souhaités auprès du public cible (Laskey, Day et Crask, 1989 


\section{BIBLIOGRAPHIE}

[1] Barasch, A, Berger, J. (2014). Broadcasting and narrowcasting : How audience size affects what people share. Journal of Marketing Research. $51: 286-299$.

[2] Berger, J. (2014). Word of mouth and interpersonal communication : A review and directions for future research. Journal of Consumer Psychology. 24 : 586-607.

[3] Berger, J, Iyengar, R. (2013). Communication channels and word of mouth : How the medium shapes the message. Journal of Consumer Research. 40 : 567-579.

[4] Chen, Z, Berger, J. (2016). How content acquisition method affects word of mouth. Journal of Consumer Research. $43: 86-102$.

[5] Hemetsberger, A. (2002). Fostering cooperation on the Internet : social exchange processes in innovative virtual consumer communities. Advances in Consumer Research. 29.

[6] Luarn, P., Lin, Y.-F., Chiu, Y.-P. (2015). Influence of Facebook brand-page posts on online engagement. Online Inf. Rev. 39, 505-519M. M. Young, The Technical Writer's Handbook. Mill Valley, CA: University Science, 1989.

[7] webster, J., \& Watson, R. T. (2002). Analyzing the past to prepare for the future: Writing a literature review. MIS Quarterly, 26(2), xiii-xxiii. 